\title{
Heaviside transform of the effective potential in the Gross-Neveu model
}

\author{
Hirofumi Yamada \\ Department of Mathematics, Chiba Institute of Technology \\ 2-1-1 Shibazono, Narashino-shi, Chiba 275 \\ Japan \\ e-mail:yamadah@cc.it-chiba.ac.jp
}

\begin{abstract}
Unconventional way of handling the perturbative series is presented with the help of Heaviside transformation with respect to the mass. We apply Heaviside transform to the effective potential in the massive Gross-Neveu model and carry out perturbative approximation of the massless potential by dealing with the resulting Heaviside function. We find that accurate values of the dynamical mass can be obtained from the Heaviside function already at finite orders where just the several of diagrams are incorporated. We prove that our approximants converges to the exact massless potential in the infinite order. Small mass expansion of the effective potential can be also obtained in our approach.
\end{abstract}




\section{Introduction}

Even if the proof of dynamical massless symmetry breaking requires genuine nonperturbative approaches, it does not necessarily mean that the perturbative expansion is totally useless. There is the possibility that non-perturbative quantities in the massless limit may be approximately calculated via perturbative approach. The purpose of this paper is to explore the possibility and show a concrete affirmative result by re-visiting the Gross-Neveu model ${ }^{1}$.

Let us consider the effective potential of the Gross-Neveu model. As is well known, ordinary massless perturbation expansion gives infra-red divergences and to cure the problem one must sum up all the one-loop diagrams. Then the summed result reveals the non-trivial vacuum configuration of $\langle\bar{\psi} \psi\rangle$ and the dynamical generation of the mass.

The point we like to note is whether such a non-perturbative effect needs, in the approximate evaluation, the infinite sum of perturbative contributions. To resolve the issue, we deal with a truncated series $V_{\text {pert }}$, without conventional loop summation, and study the approximate calculation of the effective potential $V$ at $m=0$.

A naive way of approximation would go as the following: To get around the infrared singularity we turn to the massive case and probe $V_{\text {pert }}(\sigma, m)$ at small $m$. Since the limit, $m \rightarrow 0$, cannot be taken in $V_{\text {pert }}(\sigma, m)$, we may choose some nonzero $m\left(=m^{*}\right)$ and approximate the effective potential $V(\sigma, m=0)$ by $V_{\text {pert }}\left(\sigma, m^{*}\right)$. However, the problem is that $V_{\text {pert }}(\sigma, m)$ is not valid for small enough $m$. This is the place where the Heaviside function comes in. Our suggestion to resolve the problem is to contact the Heaviside transformation of $V(\sigma, m)$ with respect to the mass ${ }^{2,3}$.

Heaviside transform of the effective potential, $\hat{V}$, is a function of $\sigma$ and $x$ which is conjugate with $m$. Then, the key relation is that $\lim _{m \rightarrow 0} V(\sigma, m)=\lim _{x \rightarrow \infty} \hat{V}(\sigma, x)$. Of course this is valid only when the both limits exist and do not apply for $V_{\text {pert }}$ and its Heaviside function, $\hat{V}_{\text {pert }}$, because those functions diverge in the limits. However 
there arises the possibility that $\hat{V}(\sigma, \infty)$ and hence $V(\sigma, 0)$ may be well approximated by putting some finite value of $x$ into $\hat{V}_{\text {pert }}$. This is because $\hat{V}_{\text {pert }}$ has the convergence radius much larger than that of $V_{\text {pert }}$. Although $\hat{V}_{\text {pert }}$ shares the similar infra-red

problems with $V_{\text {pert }}$, we will find that $\hat{V}_{\text {pert }}$ is much more convenient in this kind of massless approximation. Actually we will demonstrate that, at finite perturbative orders where just the several of Feynman diagrams are taken into account, the accurate dynamical mass is obtained via the Heaviside transform approach.

Throughout this paper, we use dimensional regularization ${ }^{4}$. We confine ourselves with the leading order of large $N$ expansion and $N$ is omitted for the sake of simplicity.

\section{Heaviside transform with respect to the mass}

In this section we summarize basic features of the Heaviside transform and illustrate our strategy by taking a simple example.

Let $\Omega(m)$ be a given function of the mass $m$. The Heaviside transform of $\Omega(m)$ is given by the Bromwich integral,

$$
\hat{\Omega}(x)=\int_{s-i \infty}^{s+i \infty} \frac{d m}{2 \pi i} \frac{\exp (m x)}{m} \Omega(m)
$$

where the vertical straight contour should lie in the right of all the possible poles and the cut of $\Omega(m) / m$ (In (1), the real parameter $s$ specifies the location of the contour). Since $\Omega(m) / m$ is analytic in the domain, $\operatorname{Re}(m)>s, \hat{\Omega}(x)$ is zero when $x<0$. It is known that the Laplace transformation (of the second kind) gives the original function as,

$$
\Omega(m)=m \int_{-\infty}^{\infty} d x \exp (-m x) \hat{\Omega}(x)
$$

Since $\hat{\Omega}(x)=0$ for $x<0$, the region of the integration effectively reduces to $[0, \infty)$. It is easy to derive the relation,

$$
\lim _{m \rightarrow+0} \Omega(m)=\lim _{x \rightarrow+\infty} \hat{\Omega}(x)
$$


where the both limits are assumed to exist. As noted before, the point of our scheme consists in utilizing $\hat{\Omega}$ to approximate the massless value of $\Omega, \Omega(0)$, by relying upon (3).

To illustrate our strategy based on (3), let us consider a simple example. Given a following truncated series in $1 / m$,

$$
f_{L}(m)=\sum_{n=0}^{L} \frac{(-1)^{n}}{m^{n+1}}
$$

we try to approximate the value of $f(m)=f_{\infty}(m)=(1+m)^{-1}$ at $m=0, f(0)=1$, by using information just contained in the truncated series (4). Since the convergence radius, $\rho$, of $f_{\infty}(m)$ is unity, we cannot have approximation better than $1 / 2$ from $f_{L}(m)$. However, the state changes if we deal with its Heaviside function.

The Heaviside transform of $f_{L}(m)$ is given by

$$
\hat{f}_{L}(x)=\sum_{n=1}^{L}(-1)^{n} \int_{s-i \infty}^{s+i \infty} \frac{d m}{2 \pi i} \frac{\exp (m x)}{m} \frac{1}{m^{n+1}}=\sum_{n=0}^{L}(-1)^{n} \frac{x^{n+1}}{(n+1) !} \theta(x),
$$

where

$$
\theta(x)= \begin{cases}1 & (x>0) \\ 0 & (x<0)\end{cases}
$$

From (5) it is easy to find that $\hat{f}(x)=\left(1-e^{-x}\right) \theta(x)$ and (3) holds for $f$ and $\hat{f}$. For our purpose it is crucial that $\rho=\infty$ for $\hat{f}_{\infty}$ while $\rho=1$ for $f_{\infty}$. The infinite convergence radius ensures us to probe the large $x$ behavior of $\hat{f}$ by $\hat{f}_{L}$ to arbitrary precision by increasing perturbative order. Due to the truncation, however, $\hat{f}_{L}$ diverges as $x \rightarrow \infty$. Then, in approximating $\hat{f}(\infty)$ and therefore $f(0)$, we stop taking the limit and input some finite value into $x$. The input value of $x$, say $x^{*}$, should be taken as large as possible in the reliable perturbative region in $x$. At this place we understand that the good convergence property of $\hat{f}_{L}$ is one of the advantage of Heaviside function.

Since the upper limit of perturbative region is not a rigorously defined concept, we determine the input value $x^{*}$ in heuristic way. Our suggestion to fix $x^{*}$ is as the following: The series (5) is valid for small $x$ but breaks down at large $x$. The breaking appears as the domination of the highest term in $\hat{f}_{L}$ which leads to the unlimited 
growth or decreasing of the function (see Fig.1). Thus $x^{*}$ is located somewhere around the beginning of the dominating behavior. Then, for odd $L$ and large even $L$, we find the plateau region just before the domination and that the region represents the end of the perturbative regime. Thus, we choose the stationary point in the plateau region as representing the typical violation of the perturbation expansion. Hence we fix $x^{*}$ by the stationarity condition,

$$
\frac{\partial \hat{f}_{L}\left(x^{*}\right)}{\partial x^{*}}=0
$$

The condition $(7)$ reads as

$$
\theta\left(x^{*}\right) \sum_{n=0}^{L} \frac{\left(-x^{*}\right)^{n}}{n !}+\delta(x) \sum_{n=0}^{L} \frac{(-1)^{n}\left(x^{*}\right)^{n+1}}{(n+1) !}=0,
$$

and reduces to

$$
\sum_{n=0}^{L} \frac{\left(-x^{*}\right)^{n}}{n !}=0
$$

The solution exists for odd $L$ and it varies with $L$. We find from (9) that the solution $x^{*}$ tends to $\infty$ as $L \rightarrow \infty$. More precisely the solution scales for large $L$ as

$$
x^{*} \sim \frac{1}{3} L
$$

We have explicitly done the numerical experiment to several higher orders and obtained the result for $L=1,5,9,13,17$,

$$
\hat{f}_{L}\left(x^{*}\right)=0.5,0.850675,0.953301,0.985166,0.995251,
$$

at $x^{*}=1,2.18061,3.33355,4.47541,5.6112$, respectively. Thus, the sequence gives good approximation of the exact value. In this toy model, one finds that $\hat{f}_{L}\left(x^{*}\right)$ converges in the $L \rightarrow \infty$ limit by using the scaling relation (10).

Up to now we have concentrated on approximating the massless value. We here point out that our scheme is capable of constructing the small $m$ expansion of $\Omega(m)$, that is, the scheme allows the approximation of the function itself when $m$ is small. Consider in general the approximation of the derivatives at $m=0$,

$$
\Omega^{(k)}(0)=\left.\frac{\partial^{k} \Omega}{\partial m^{k}}\right|_{m=0}
$$


which is needed when one constructs the small $m$ expansion of $\Omega(m)$,

$$
\Omega(m)=\Omega(0)+\frac{\Omega^{(1)}(0)}{1 !} m+\frac{\Omega^{(2)}(0)}{2 !} m^{2}+\cdots .
$$

The coefficients, $\Omega^{(k)}(0)(k=1,2,3, \cdots)$, can be approximated as follows. The starting formula is that

$$
\Omega^{(k)}(m) \stackrel{\mathcal{H}}{\rightarrow} \int_{-\infty}^{x} d t(-t)^{k} \frac{\partial \hat{\Omega}(t)}{\partial t}(x)
$$

where we used

$$
m \frac{\partial \Omega}{\partial m} \stackrel{\mathcal{H}}{\rightarrow}-x \frac{\partial \hat{\Omega}(x)}{\partial x}, \quad \frac{1}{m} \Omega \stackrel{\mathcal{H}}{\rightarrow} \int_{-\infty}^{x} d t \hat{\Omega}(t) .
$$

Here $\mathcal{H}$ above the arrow represents the Heaviside transformation. Hence, from the agreement condition (3) we find

$$
\lim _{m \rightarrow 0} \Omega^{(k)}(m)=\lim _{x \rightarrow \infty} \alpha_{k}(x)=\int_{-\infty}^{\infty} d t(-t)^{k} \frac{\partial \hat{\Omega}(t)}{\partial t} .
$$

Now in our perturbative approach we use $\Omega_{L}$ (the truncated series at the order $L$ ) for real $\Omega$. Then, we show that we can simulate $\alpha_{k}(\infty)$ by $\alpha_{k}\left(x_{k}^{*}\right)$ where $x_{k}^{*}$ may be fixed following the same reasoning we presented for the case of $f(0)$ approximation. Namely we guess that the break down of the perturbative expansion is represented by the plateau region, if it exists, just before the unlimited growth of the size of the function. Therefore we use stationarity condition which reads,

$$
\left.\frac{\partial \alpha_{k}}{\partial x}\right|_{x=x_{k}^{*}}=\left(-x_{k}^{*}\right)^{k} \frac{\partial \hat{\Omega}_{L}}{\partial x_{k}^{*}}=0,
$$

and find that $x_{k}^{*}$ satisfies the same condition as that for $x^{*}$. Thus the solution of (17) is universal for all $k$ and fixes the coefficients of the small $m$ expansion to all orders. This is desirable since the uncertainty connected with the choice of $x^{*}$ and $x_{k}^{*}$ is minimized. We note that for $\alpha_{k}\left(x^{*}\right)(k=1,2,3, \cdots)$ the integration is necessary and $\theta(x)$ and $\delta$ functions should be kept in the integrand in general.

As an example let us calculate the small $m$ expansion of $f(m)$. The coefficient $\alpha_{k}$ is given at order $L$ by,

$$
\alpha_{k}=(-1)^{k} \sum_{n=0}^{L} \frac{(-1)^{n} x^{k+n+1}}{n !(k+n+1)} .
$$


By substituting $x^{*}$ at $L=17$ into $\alpha_{k}$, we have the following satisfactory approximant of $f(m)=1-m+m^{2}-\cdots$;

$$
0.995251-0.970003 m+0.90271 m^{2}-0.78284 m^{3}+0.62233 m^{4}+\cdots .
$$

\section{Application to the effective potential}

Having prepared basic analysis, we turn to a model field theory which is of our main interest. Consider the Gross-Neveu model at the leading order of large $N$ expansion ${ }^{1}$. The Lagrangian is given within dimensional regularization ${ }^{4}$ at $D=4-2 \epsilon$ by

$$
\begin{aligned}
\mathcal{L} & =\bar{\psi}\left(i \gamma^{\mu} \partial_{\mu}-m\right) \psi-\frac{1}{2} \sigma^{2}-\frac{g}{\sqrt{N}} \mu^{\epsilon} \sigma \bar{\psi} \psi+\mathcal{L}_{c t}, \\
\mathcal{L}_{c t} & =A \sigma-B \frac{1}{2} \sigma^{2}
\end{aligned}
$$

where

$$
\psi=\left(\psi_{1}, \cdots, \psi_{N}\right), \quad A=-\frac{\sqrt{N} m g \mu^{-\epsilon}}{2 \pi} \frac{\hat{1}}{\epsilon}, \quad B=\frac{g^{2}}{2 \pi} \frac{\hat{1}}{\epsilon}, \quad \frac{\hat{1}}{\epsilon}=\frac{1}{\epsilon}-\gamma+\log (4 \pi) .
$$

Here $\overline{M S}$ scheme $^{5}$ was used for the subtraction. It is well known that the model generates the dynamical fermion mass, $m_{d y n}=\Lambda$, where $\Lambda$ denotes the renormalization group invariant scale in $\overline{M S}$ scheme.

At the leading order of $1 / N$ expansion, the effective potential is given by the sum of diagrams shown in Fig.2. The straightforward calculation gives

$V(\sigma, m)=\frac{m^{2}}{4 \pi}\left(\log \frac{m^{2}}{\mu^{2}}-1\right)+\frac{m g \sigma}{2 \pi} \log \frac{m^{2}}{\mu^{2}}+\frac{g^{2} \sigma^{2}}{4 \pi}\left(\log \frac{m^{2}}{\Lambda^{2}}+2\right)-\sum_{n=3}^{\infty} \frac{(-g \sigma)^{n}}{\pi n(n-1)(n-2)} m^{-n+2}$.

We note that although the naive power counting with respect to $N$ leads that the contribution with many $\sigma$-legs corresponds to higher order in $1 / N$, they must be included since the vacuum value of $\sigma$ is of order $\sqrt{N}$.

The series (22) converges only when $|g \sigma / m|<1$ and hence the small $m$ behavior relevant to the dynamical mass generation is not known from (22). However, Heavi- 
side transformation enlarges the convergence radius and enables us to study the large $x$ behavior of the corresponding Heaviside function, $\hat{V}(\sigma, x)$, as we can see below.

To obtain $\hat{V}(\sigma, x)$ we need to know the transform of $m^{k}(k=0,1,2, \cdots), m \log m, m^{2} \log m$ and $1 / m^{k}$. Here the following formula is basic,

$$
m \Omega(m) \stackrel{\mathcal{H}}{\rightarrow} \frac{\partial \hat{\Omega}(1 / x)}{\partial x} .
$$

For example from (1) we have

$$
\log (m) \stackrel{\mathcal{H}}{\rightarrow}(-\gamma-\log (x)) \theta(x)
$$

The use of (23) on (24) then leads to

$$
\begin{aligned}
m \log m & \stackrel{\mathcal{H}}{\rightarrow}-\frac{1}{x} \theta(x)-(\gamma+\log x) \delta(x), \\
m^{2} \log m & \stackrel{\mathcal{H}}{\rightarrow} \frac{1}{x^{2}} \theta(x)-\frac{2}{x} \delta(x)-(\gamma+\log x) \delta^{\prime}(x) .
\end{aligned}
$$

The transformation of $m^{k}$ is easily obtained from

$$
1 \stackrel{\mathcal{H}}{\rightarrow} \theta(x)
$$

as

$$
m^{k} \stackrel{\mathcal{H}}{\rightarrow} \delta^{(k-1)}(x) \quad(k=1,2,3, \cdots)
$$

The $\delta$ functions are needed when one carries out Laplace integrals for the Heaviside functions. This is because the $\delta$ function terms cancel out the divergences coming from the first terms of (25) and (26), for example. Since the integration over $x$ is however not necessary as long as the approximation in the massless limit is concerned, we omit, for a while, $\delta$ functions and set $\theta(x)=1$ in the transformed functions.

Now, using the results, (24), (25), (26), (27), (28) and $\mathcal{H} m^{-k}=x^{k} / k$ !, we find

$$
\hat{V}(\sigma, x)=\frac{1}{2 \pi x^{2}}-\frac{g \sigma}{\pi x}+\frac{g^{2} \sigma^{2}}{2 \pi}(-\log \Lambda x-\gamma+1)-\sum_{n=3}^{\infty} \frac{(-g \sigma)^{n} x^{n-2}}{\pi n !(n-2)} .
$$

Note that, due to the creation of $1 / k$ ! in $\mathcal{H}\left[1 / m^{k}\right]$, the series converges for any large $x$. Therefore the large $x$ behavior of $\hat{V}$ can be easily accessed by increasing the order of expansion. This is one of the advantages of $\hat{V}$ over $V$. 
We turn to the approximation of the massless effective potential by perturbative series at order $L, \hat{V}_{L}$. At $L$-th order, we have just first $L+1$ terms of (29) and find

$$
\hat{V}_{L}(\sigma, x)=\frac{1}{2 \pi x^{2}}-\frac{g \sigma}{\pi x}+\frac{g^{2} \sigma^{2}}{2 \pi}(-\log \Lambda x-\gamma+1)-\sum_{n=3}^{L} \frac{(-g \sigma)^{n} x^{n-2}}{\pi n !(n-2)} .
$$

The input $x^{*}$ will be determined as in the previous section. Actually the break down appears as the domination of the last term in $\hat{V}_{L}$ which shows up as its unlimited behavior for large $x$. This can be seen in Fig. 3. And before the domination the function experiences a stationary behavior for odd $L$ and large even $L$. We find that the plateau region represents the end of the reliable perturbative regime and thus we fix $x^{*}$ by the equation,

$$
\frac{\partial \hat{V}_{L}\left(\sigma, x^{*}\right)}{\partial x^{*}}=0
$$

If there are several solutions we should input the largest one into $x^{*}$ due to the obvious reason.

Now, the condition (31) gives $x^{*}$ as constant/go for odd $L{ }^{\dagger}$. For odd $L$, the substitution of the solution into $\hat{V}_{L}$ gives the optimized potential $V_{\text {opt }}(\sigma)$. For example for $L=3$, we have the solution, $g \sigma x^{*}=1.59607$, and this gives the optimized potential,

$$
V_{\text {opt }}=\frac{g^{2} \sigma^{2}}{2 \pi}\left(\log \frac{g \sigma}{\Lambda}-0.373264\right) \text {. }
$$

The dynamical mass is given from $V_{\text {opt }}$ in the standard way. Note that, since $x$ must be positive (see section 2), the region of $V_{\text {opt }}$ thus approximated is restricted to the positive $\sigma$. In the following, we summarize the result of the approximate calculation of the dynamical mass for $L=3,5,7,9,11$;

$$
\begin{aligned}
& L=3, \quad m_{d y n} / \Lambda=0.880966 \quad\left(\text { at } g \sigma x^{*}=1.59607\right) \\
& L=5, \quad m_{d y n} / \Lambda=0.97760 \quad\left(\text { at } g \sigma x^{*}=2.18061\right) \\
& L=7, \quad m_{d y n} / \Lambda=0.99401 \quad\left(\text { at } g \sigma x^{*}=2.75900\right)
\end{aligned}
$$

${ }^{\dagger}$ For even $L$, there is no solution for (31). In these cases, however, the use of $\partial^{2} \hat{V}_{L}(\sigma, x) / \partial x^{2}=0$ gives a solution by which we still have good approximation of $m_{d y n}$ at $L=4,6,8, \cdots$. 


$$
\begin{gathered}
L=9, \quad m_{d y n} / \Lambda=0.99809 \quad\left(\text { at } g \sigma x^{*}=3.33355\right) \\
L=11, \quad m_{d y n} / \Lambda=0.999326 \quad\left(\text { at } g \sigma x^{*}=3.90545\right)
\end{gathered}
$$

The above result is quite good. Thus, via Heaviside transform approach, the dynamical mass is approximated only from perturbative information.

The small mass expansion can be also obtained. Our task is just to substitute the solution of (31) into the approximate coefficients,

$$
\alpha_{k}\left(x^{*}\right)=\int_{-\infty}^{x^{*}} d x(-x)^{k} \frac{\partial \hat{V}_{L}(\sigma, x)}{\partial x}
$$

To perform integration, we need the full form of $\hat{V}_{L}$ including the $\theta$ and $\delta$ functions. The full form is given by

$$
\begin{aligned}
\hat{V}_{L}(x) & =\frac{1}{2 \pi}\left[\frac{1}{x^{2}} \theta(x)-\frac{2}{x} \delta(x)-(\gamma+\log x \mu+1 / 2) \delta^{\prime}(x)\right]-\frac{g \sigma}{\pi}\left[\frac{1}{x} \theta(x)+(\gamma+\log x \mu) \delta(x)\right] \\
& -\frac{g^{2} \sigma^{2}}{2 \pi}(\gamma+\log x \Lambda-1) \theta(x)-\sum_{n=3}^{L} \frac{(-g \sigma)^{n} x^{n-2}}{\pi n !(n-2)} \theta(x) .
\end{aligned}
$$

From $(35) \alpha_{k}$ is given at $L$-th order as

$$
\begin{aligned}
& \alpha_{1}=\frac{g \sigma}{\pi}\left[-\frac{1}{X}+1-\gamma+\log (g \sigma / \mu)-\log X+\sum_{n=2}^{L} \frac{(-1)^{n} X^{n-1}}{n !(n-1)}\right] \\
& \alpha_{2}=\frac{1}{\pi}\left[\log (g \sigma / \mu)-\log X+2+\sum_{n=1}^{L} \frac{(-1)^{n} X^{n}}{n ! n}\right] \\
& \alpha_{k}=\frac{(-1)^{k+1}}{\pi(g \sigma)^{k-2}} \sum_{n=0}^{L} \frac{(-1)^{n} X^{n+k-2}}{n !(n+k-2)} \quad(k>2),
\end{aligned}
$$

where $X=x^{*} g \sigma$. At $L=11$, for example, we have

$$
\begin{aligned}
V(\sigma, m) & \sim \frac{g^{2} \sigma^{2}}{2 \pi}\left(\log \frac{g \sigma}{\Lambda}-0.499326\right)+m \frac{g \sigma}{\pi}(\log g \sigma-0.00139409)+\frac{m^{2}}{2} \frac{1}{\pi}(\log g \sigma+1.00595) \\
& +\frac{m^{3}}{6 g \sigma} 0.973714-\frac{m^{4}}{24 g^{2} \sigma^{2}} 0.878954+O\left(m^{5}\right)
\end{aligned}
$$

This is quite accurate because the exact result reads

$V(\sigma, m)=\frac{g^{2} \sigma^{2}}{2 \pi}\left(\log \frac{g \sigma}{\Lambda}-\frac{1}{2}\right)+\frac{1}{\pi} m g \sigma \log \frac{g \sigma}{\mu}+\frac{m^{2}}{2 \pi}\left(\log \frac{g \sigma}{\mu}+1\right)+\frac{m^{3}}{6 g \sigma}-\frac{m^{4}}{24 g^{2} \sigma^{2}}+O\left(m^{5}\right)$.

Before closing this section, we prove that our approximants for the massless potential converges to the exact result in the $L \rightarrow \infty$ limit. That is, $\lim _{L \rightarrow \infty} \hat{V}_{L}\left(\sigma, x^{*}\right)=$ 
$V(\sigma, 0)$. From (30) we find that $\lim _{L \rightarrow \infty} \partial \hat{V}_{L} / \partial x$ can be easily summed up and, using $\lim _{L \rightarrow \infty} \hat{V}_{L}=\hat{V}$ (given by $(29)$ ),

$$
\frac{\partial \hat{V}(\sigma, x)}{\partial x}=-\frac{1}{\pi x^{3}} \exp [-g \sigma x] .
$$

The perturbative truncation of $(39)$ is given by expanding $\exp [-g \sigma x]$ to relevant orders. Since $\rho=\infty$ for the series expansion of (39), the solution of the truncated version of $(39)=0$ approaches to $\infty$ in the $L \rightarrow \infty$ limit. More precisely we find the scaling of the solution for large $L$,

$$
g \sigma x^{*} \sim \frac{1}{3} L
$$

Now consider the reminder $\hat{R}_{L}$, defined by

$$
\hat{R}_{L}=-\sum_{n=L+1}^{\infty} \frac{(-g \sigma)^{n}}{\pi n !(n-2)} x^{n-2} .
$$

Since $\hat{V}_{L}+\hat{R}_{L}=\hat{V}_{\infty}$ and $\rho=\infty$ for $\hat{V}_{\infty}$, it is sufficient to show that

$$
\hat{R}_{L}\left(\sigma, x^{*}\right) \rightarrow 0 \quad(L \rightarrow \infty)
$$

Note here that $x^{*}$ depends on $L$ and behaves at large $L$ as (40). Now, using the Stirling's formula and (40), we have

$$
\left|\hat{R}_{L}\right|<\frac{1}{x^{*} \sqrt{2 \pi^{3} L^{3}}} \frac{\left(e g \sigma x^{*} / L\right)^{L+1}}{1-e g \sigma x^{*} / L}<\frac{9(g \sigma)^{2}}{\sqrt{2 \pi^{3}}(3 / e-1)} L^{-7 / 2}(e / 3)^{L} \rightarrow 0 \quad(L \rightarrow \infty),
$$

which proves the convergence.

\section{Discussion}

One reason of the success of our approximate calculation is that the transformed function has infinite radius of convergence. The other reason is that, as the order

increases, $\hat{V}_{L}(\sigma, x)$ quickly approaches to the value at $x=\infty$ for fixed $\sigma$. If one uses the closed form,

$$
\hat{V}=\frac{g^{2} \sigma^{2}}{2 \pi}\left(\log \frac{g \sigma}{\Lambda}-\frac{1}{2}\right)+\frac{g^{2} \sigma^{2}}{\pi} \int_{g \sigma x}^{\infty} d x \frac{e^{-x}}{x^{3}}
$$


one finds the reason by expanding (44) for large $g \sigma x$,

$\hat{V}(\sigma, x)=\frac{g^{2} \sigma^{2}}{2 \pi}\left(\log \frac{g \sigma}{\Lambda}-\frac{1}{2}\right)+\frac{1}{\pi x^{2}} \exp [-g \sigma x]\left(\frac{1}{g \sigma x}+(-3)\left(\frac{1}{g \sigma x}\right)^{2}+(-3)(-4)\left(\frac{1}{g \sigma x}\right)^{3}+O\left(\left(\frac{1}{g \sigma x}\right)^{4}\right)\right)$.

By contrast, the original function has the power-like expansion as shown in (38). Thus it is obvious that the approximation of the massless potential is more convenient in $\hat{V}_{L}$ since it approaches to the "massless" value much faster than $V_{L}$. The reason behind why the transformed function behaves so good is not known to us.

We have shown that the perturbative series at finite orders produces the approximate massless effective potential and the dynamical mass. The deformation of the effective potential was made by Heaviside transform with respect to the mass and the stationarity prescription to fix the input value $x^{*}$ has found to work good. It was also shown that our scheme is capable of approximating the small $m$ behavior. Thus the Heaviside transform drastically improves the status of the perturbative approximation of physical quantities. We are under the study of full approximation method by which the general case where the explicit mass is not small can be treated. The result of investigation will be reported elsewhere.

\section{Acknowledgment}

The author thanks Dr. H. Suzuki for the critics on the primitive version of this work and stimulating discussion. 


\section{References}

1 D.J.Gross and A.Neveu, Phys. Rev. D10 (1974) 3235.

2 S. Moriguchi et al, Suugaku Koushiki II, Iwanami Shoten (in Japanese).

3 H. Yamada, Mod. Phys. Lett. A11 (1996) 1001.

4 G. t'Hooft and M. Veltman, Nucl. Phys. B44 (1972) 189;

C. G. Bollini and J. J. Giambiagi, Phys. Lett. B40 (1972) 566;

G. M. Cicuta and E. Montaldi, Nuovo Cimento Lett. 4 (1972) 329.

5 W. A. Bardeen, A. J. Buras, D. W. Duke and T. Muta, Phys. Rev. D18 (1978) 3998.

\section{Figure Captions}

Figure 1 Model series $\hat{f}_{L}(x)$ is shown for $L=1,6,11$.

Figure 2 The Feynman diagrams contributing to the effective potential at the leading order of $1 / N$ expansion.

Figure 3 The Heaviside functions of the effective potential with fixed $\sigma$ at $L=3,6$ and 9 . For the sake of the simplicity, we have set that $g \sigma=1$ and $\Lambda=1$. 